\title{
Extremal black holes and the limits of the third law
}

\author{
Stefano Liberati*, Tony Rothman ${ }^{\dagger}$ and Sebastiano Sonego ${ }^{\ddagger}$ \\ * International School for Advanced Studies, via Beirut 2-4, Trieste 34014, Italy; \\ INFN sezione di Trieste. \\ ${ }^{\dagger}$ Dept. of Physics, Illinois Wesleyan University, Bloomington, IL 61702, USA. \\ $\ddagger$ Università di Udine, Via delle Scienze 208, 33100 Udine, Italy.
}

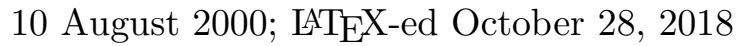

Paper awarded of an "honorable mention" in the Annual Competition

of the Gravity Research Foundation for the year 2000.

\begin{abstract}
Recent results of quantum field theory on a curved spacetime suggest that extremal black holes are not thermal objects and that the notion of zero temperature is ill-defined for them. If this is correct, one may have to go to a full semiclassical theory of gravity, including backreaction, in order to make sense of the third law of black hole thermodynamics. Alternatively it is possible that we shall have to drastically revise the status of extremality in black hole thermodynamics.
\end{abstract}

Since the early 1970s, relativists have recognized that a remarkable analogy exists between the three laws of thermodynamics and the behavior of black holes, behavior that is encoded in the three laws of black hole dynamics. Indeed, since Hawking's celebrated discovery of black hole radiation [罒], the general consensus has been that the laws of black hole dynamics are no mere analogy, as remarkable as the analogy may be, but precisely the laws of thermodynamics applied to black holes. Historically, most research has focused on the first and second laws, which explicitly identify the surface area of a black hole with its entropy. In the past few years, however, increasing attention has been paid to the third law, and it may be the third law that is pointing toward the limits of black hole thermodynamics as currently understood.

The third law of ordinary thermodynamics admits two different formulations, both due to Nernst. The "entropic" formulation states that the entropy of a system approaches a constant, independent of all the macroscopic parameters, as the temperature approaches absolute zero. According to the Planck postulate, this constant can be set equal to zero. The second formulation, the so-called "unattainability" law, states that it is impossible to reach absolute zero in a finite number of steps. For common physical systems the

*liberati@sissa.it

$\dagger$ trothman@titan.iwu.edu

${ }^{\ddagger}$ sebastiano.sonego@uniud.it 
two versions are equivalent, although one can imagine situations in which the equivalence breaks down [2].

For a black hole with mass $M$, charge $Q$ and specific angular momentum $a$, the temperature is proportional to the surface gravity $\kappa \propto\left(M^{2}-Q^{2}-a^{2}\right)^{1 / 2}$, and the entropy, according to the Bekenstein-Hawking formula, is simply one-fourth the area of the event horizon. Clearly, the temperature vanishes when $M^{2}=Q^{2}+a^{2}$, the "extremal" limit. However, the area still depends on the parameters $Q$ and $a$ as $\kappa \rightarrow 0$, which represents an obvious violation of the entropic formulation of the third law. On the other hand, it is now well known that in black hole dynamics one cannot reach the extremal state by any continuous, finite-time process [3]; therefore the unattainability formulation of the third law appears favored.

Unfortunately, direct evaluations of the entropy do not all lead to the same results: The current status can be broadly summarized as follows: Semiclassical calculations, which take into account the peculiar global structure of the extremal spacetime manifold, lead to a zero value for the entropy of extremal black holes [4, 5, 6, 7, 8]. On the other hand, string theory calculations, in which the entropy can be explained on purely statistical grounds, find that extremal solutions obey the Bekenstein-Hawking formula (see [9] for a comprehensive review). Both sets of results, however, imply a violation of the entropic formulation of the third law. Indeed, even if extremal black holes had a vanishing entropy, zero is not the value to which the entropy of nearly extremal black holes tends in the limit $\kappa \rightarrow 0$. This lack of a good limit hints at the existence of a discontinuity in the thermodynamical behaviour of the two classes of objects.

Such considerations suggest that one should focus on the thermodynamic nature of the extremal black holes, rather than try to fix the entropic formulation of the third law [10]. Along these lines, we have studied the thermal nature of an "incipient" extremal black hole - a collaping spherical body with an exterior extremal Reissner-Nordström (RN) metric [11].

The main result of our investigation is that incipient extremal black holes do not behave as thermal objects at any time during their history. In other words, they do not radiate as black bodies and their emission spectrum, as well as the expectation value and variance of their stress-energy-momentum tensor, do not correspond, at any finite time, to the limit for $\kappa \rightarrow 0$ of the non-extremal incipient black holes.

Then one can conclude that neither temperature nor thermodynamic entropy can be associated with them. This conclusion evidently would lead to an explanation of the third law of black hole thermodynamics in a radical way: The temperature of a black hole cannot be reduced to zero because no black hole zero temperature state exists.

The steps toward demonstrating that such incipient extremal RN black holes have no thermal properties are reasonably straightforward and can be summarized here. (For a few details see the appendix and for a full derivation see [11.) One employs the socalled "moving mirror" analogy to model the collapsing body by a one-dimensional mirror moving in two-dimensional Minkowski space [12, 13]. On the background we assume a propagating quantum scalar field. Incoming scalar rays, passing through the center of the star are reflected off the mirror to become outgoing rays. Due to the motion of the mirror, one expects the In and Out vacuum states to differ, leading to particle production at late times (Hawking radiation). The first task, then, is to calculate the late-time history of the mirror, which coincides with the worldine of the star's center written in 
Eddington-Finkelstein null coordinates.

The calculation is reasonably straightforward if one carries out a few intermediate steps in Kruskal coordinates. Unfortunately, Kruskal coordinates become ill-defined on the horizon in the extremal limit. Nevertheless, we have found a generalization of the Kruskal transformation that allows us to calculate the late-time worldline of the star's centre all the way to the horizon. Remarkably, this trajectory turns out to correspond to that of a uniformly accelerated mirror in Minkowski spacetime. The particle spectrum for the uniformly accelerating mirror can be found by calculating the Bogoliubov coefficients, which relate the In and Out vacua. These are known to be proportional to the $K_{1}$ Bessel function [12, 13]. Consequently, the spectrum is non-Planckian, and no temperature can be assigned to the object at any time during its history, in contrast to the situation regarding non-extremal incipient black holes.

Apart from its nonthermal character, the most striking aspect of the extremal black hole spectrum is that its amplitude contains a lumped constant $A$ that depends on the history of the collapsing object. At first sight this appears to be a direct violation of the no-hair theorems. Furthermore, given that the object is an extremal one $\left(Q^{2}=M^{2}\right)$, because it is radiating neutral particles, the mass will decrease relative to the charge, leaving a naked singularity as the final product of collapse, in apparent violation of the cosmic censorship hypothesis.

We have, however, examined the behavior of the stress-energy-momentum tensor and its variance for these extremal incipient black holes. For the uniformly accelerated mirror, the flux is known to exhibit certain pathologies: Its expectation value is zero, despite the fact that particles are definitely being emitted, as demonstrated by the nonzero Bogoliubov coefficients. That the expectation value of the flux vanishes saves the cosmic censorship hypothesis because, although particles are created, the average energy emitted is zero. (This counter-intuitive result is related to the fact that the $K_{1}$ Bessel function, and hence the spectrum, diverges in the zero energy limit, so a detector is overwhelmed by soft scalar particles.) We find, moreover, that the variance of the flux, which also contains the constant $A$, is nonzero but vanishes as a power law at late times. On the one hand, the fact that the expectation value of the flux is zero and its variance does vanish at late time saves the no-hair theorem because any measurement of the object's history becomes progressively more difficult. On the other hand, that the decay of the variance obeys a power law is a further proof that extremal black holes are qualitatively different from non-extremal ones, for which the variance of the flux vanishes exponentially with time.

Although the former results are valid just for incipient black holes, one can take the as hints towards more general conclusions, we can then conjecture that all extremal black holes are not thermal objects; more precisely, the set of thermodynamical states of the black holes does not include extremal ones. An alternative possibility, however, is that the test field approximation used in all calculations of this sort is inappropriate when dealing with such delicate issues, and that one must go to a more sophisticated theory. The divergence in the particle spectrum of the uniformly accelerated mirror, for example, is reminiscent of the typical infrared catastrophe of QED, which manifests itself in the process of Bremsstrahlung [14]. Just as those divergences are removed by considering the recoil of electrons, it might be necessary to consider recoil of the moving mirror under the action of the scalar field [15]. Nevertheless, it is far from obvious that including such 
radiative corrections for the mirror could be transplanted in any straightforward way to the case of an incipient black hole, and it is equally unobvious that it would transform the Bessel-like spectrum into a thermal one. Taking backreaction into account would in fact be an admission that the test-field approximation employed is never valid and would be tantamount to a confession that a more sophisticated theory is indeed necessary in order to assess the true meaning of the third law of black hole dynamics.

It may be that even the inclusion of backreaction will prove insufficient to impart thermal properties to extremal black holes. Simultaneously with our work, Anderson, Hiscock and Taylor [16] have demonstrated that for static RN geometries, zero-temperature black holes cannot exist if one considers spacetime perturbations due to the backreaction of quantum fields. Thus, at the current state of knowledge it appears that either extremal black holes are simply not thermodynamic objects, or they represent solutions in which the external field approach to semiclassical gravity breaks down. In either case they not only differ dramatically from their non-extremal counterparts but certainly represent the limit of current theory.

Note added. The conclusions discussed here for extremal Reissner-Nordström black holes evidently pertain to extremal Kerr black holes as well. See [18].

\section{Appendix: Asymptotic worldlines for incipient extremal black holes}

To derive the late-time worldline for the centre of a body collapsing to an extremal black hole, we start with the usual RN geometry in the case $Q^{2}=M^{2}$,

$$
\mathrm{d} s^{2}=-\left(1-\frac{M}{r}\right)^{2} \mathrm{~d} t^{2}+\left(1-\frac{M}{r}\right)^{-2} \mathrm{~d} r^{2}+r^{2} \mathrm{~d} \Omega^{2},
$$

where $\mathrm{d} \Omega^{2}$ is the metric on the unit sphere. This line element, however, is singular on the horizon $(r=M)$, so we want to transform to Kruskal coordinates. Normally, one does this by first constructing null coordinates $u=t-r_{*}$ and $v=t+r_{*}$, then going to Kruskal coordinates $\mathcal{U}$ and $\mathcal{V}$ which are regular and finite on the horizon. (Here $r_{*}$ is the usual tortoise coordinate.) However, the usual Kruskal transformation is $\mathcal{U}=-e^{-\kappa u}$ and $\mathcal{V}=e^{\kappa v}$. Because $\kappa$ vanishes in the limit of extremality, the Kruskal transformation results in constant values of $\mathcal{U}$ and $\mathcal{V}$, which are thus ill-defined in that limit.

This situation can be remedied, however, by a simple generalization of the Kruskal transformation. If one defines $\mathcal{U}$ and $\mathcal{V}$ through

$$
\left.\begin{array}{l}
u=-\psi(-\mathcal{U}) \\
v=\psi(\mathcal{V})
\end{array}\right\}
$$

where

$$
\psi(\xi)=4 M\left(\ln \xi-\frac{M}{2 \xi}\right),
$$

then it is not difficult to show that the extremal RN metric (A.1) can be rewritten in terms of $\mathcal{U}$ and $\mathcal{V}$ as

$$
\mathrm{d} s^{2}=-\frac{(r-M)^{2}}{r^{2}} \psi^{\prime}(-\mathcal{U}) \psi^{\prime}(\mathcal{V}) \mathrm{d} \mathcal{U} \mathrm{d} \mathcal{V}+r^{2} \mathrm{~d} \Omega^{2}
$$


This metric is apparently degenerate on the horizon, but in fact near $r=M$ we have $\mathcal{U}=-\psi^{-1}(-u) \sim-\psi^{-1}(\psi(r-M))=-(r-M)$ and

$$
\psi^{\prime}(-\mathcal{U}) \sim \frac{4 M}{r-M}+\frac{2 M^{2}}{(r-M)^{2}} \sim \frac{2 M^{2}}{(r-M)^{2}} .
$$

Furthermore, since $\mathcal{V}$ is everywhere nonzero and finite then $\psi^{\prime}(\mathcal{V})$ is regular there. Now the form taken by the metric (A.4) is asymptotically

$$
\mathrm{d} s^{2} \sim-\frac{2 M^{2}}{r^{2}} \psi^{\prime}(\mathcal{V}) \mathrm{d} \mathcal{U} \mathrm{d} \mathcal{V}+r^{2} \mathrm{~d} \Omega^{2}
$$

because the $(r-M)^{2}$ in the numerator of Eq. (A.4) is killed by the $(r-M)^{2}$ in the denominator of Eq. (A.5). Consequently, $\mathcal{U}$ and $\mathcal{V}$ are good Kruskal-like coordinates.円

The next step is to use this result to calculate the asymptotic worldline of the centre of the star. First, we match the coordinates $\mathcal{U}$ and $\mathcal{V}$ onto interior null coordinates, say, $U$ and $V$. In particular, if two nearby outgoing rays differ by $\mathrm{d} U$ inside the star, then they will differ by $\mathrm{d} U=\beta(\mathcal{U}) \mathrm{d} \mathcal{U}$, with $\beta$ a regular function, outside. Similarly, $\mathrm{d} V=\zeta(v) \mathrm{d} v$, where $\zeta$ is another regular function. Then if $v=\bar{v}$ represents the last ray that passes through the center of the star before the formation of the horizon, to first order we have $\mathrm{d} V=\zeta(\bar{v}) \mathrm{d} v$, where $\zeta(\bar{v})$ is constant.

We can write near the horizon

$$
\mathrm{d} U=\beta(0) \frac{\mathrm{d} \mathcal{U}}{\mathrm{d} u} \mathrm{~d} u
$$

Since for the center of the star $\mathrm{d} U=\mathrm{d} V=\zeta(\bar{v}) \mathrm{d} v$, this immediately integrates to

$$
\zeta(\bar{v})(v-\bar{v})=\beta(0) \mathcal{U}(u)=-\beta(0) \psi^{-1}(-u) \sim-2 \beta(0) \frac{M^{2}}{u} .
$$

The last approximation follows from Eq. (A.3) where $\xi \sim \psi^{-1}\left(-2 M^{2} / \xi\right)$ near the horizon.

Thus the late-time worldline for the center of the star is, finally, represented by the equation

$$
v \sim \bar{v}-\frac{A}{u}, \quad u \rightarrow+\infty
$$

where $A=2 \beta(0) M^{2} / \zeta(\bar{v})$ is a positive constant that depends on the dynamics of collapse. This is a hyperbolic trajectory, equivalent to that of a uniformly accelerated mirror in two-dimensional Minkowski space. From this point on, one can use results already in the literature, which establish that the particle production in such a scenario is nonthermal.

\footnotetext{
${ }^{1}$ Double-null coordinates that are regular on general degenerate Killing horizons have been constructed by Lake 17. In the present case, Lake's coordinates would essentially correspond with keeping only the last term inside brackets in Eq. A.3. We prefer to use instead the function $\psi$ given by Eq. A.3) because it allows us to define coordinates $\mathcal{U}$ and $\mathcal{V}$ that cover not only a region near the horizon, but the whole exterior spacetime. Thus, our coordinates $\mathcal{U}$ and $\mathcal{V}$ can be regarded as a deformation of Lake's double-null coordinates, with the difference becoming relevant far from the horizon.
} 
Extremal black holes...

\section{References}

[1] S. W. Hawking, Commun. Math. Phys. 43, 199 (1975).

[2] P.T. Landsberg, Thermodynamics and Statistical Mechanics (Dover, New York, 1990).

[3] W. Israel, Phys. Rev. Lett. 57, 397 (1986).

[4] G. Gibbons and R. Kallosh, Phys. Rev. D 51, 2839 (1995).

[5] S. W. Hawking, G. Horowitz, and S. F. Ross, Phys. Rev. D 51, 4302 (1995).

[6] S. W. Hawking and G. Horowitz, Class. Quantum Grav. 13, 1487 (1996).

[7] S. Liberati and G. Pollifrone, Phys. Rev. D 56, 6458 (1997).

[8] K. Kiefer and J. Louko, Ann. Phys. (Leipzig) 8, 67 (1999).

[9] G. T. Horowitz, "Quantum states of black holes," gr-qc/9704072.

[10] R. Wald, Phys. Rev. D 56, 6467 (1997).

[11] S. Liberati, T. Rothman, and S. Sonego, "Nonthermal nature of incipient extremal black holes," gr-qc/0002019.

[12] P. C. W. Davies and S. A. Fulling, Proc. R. Soc. Lond. A 356, 237 (1977).

[13] N. D. Birrell and P. C. W. Davies, Quantum Fields in Curved Space (Cambridge University Press, Cambridge, 1982).

[14] F. Mandl and G. Shaw, Quantum Field Theory (Wiley, Chichester, 1984).

[15] R. Parentani, Nucl. Phys. B465, 175 (1996).

[16] P. R. Anderson, W. H. Hiscock, and B. E. Taylor, "Do semiclassical zero temperature black holes exist?" gr-qc/0002007.

[17] K. Lake, Phys. Rev. D 20, 370 (1979).

[18] T. Rothman Phys. Lett. A (to appear); gr-qc/0006036. 